\title{
Symmetries in peripheral ocular aberrations
}

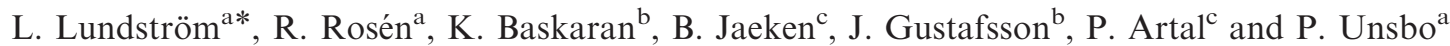 \\ ${ }^{a}$ Biomedical and X-ray Physics, Royal Institute of Technology (KTH), Stockholm, Sweden; ${ }^{b}$ Section of Optometry \\ and Vision Science, Linnaeus University, Kalmar, Sweden; ${ }^{c}$ Laboratorio de Optica (LOUM), University of Murcia, \\ Murcia, Spain
}

(Received 28 November 2010; final version received 14 February 2011)

\begin{abstract}
A mirror symmetry in the aberrations between the left and right eyes has previously been found foveally, but while a similar symmetry for the peripheral visual field is likely, it has not been investigated. Nevertheless, the peripheral optical quality is often evaluated in only one eye, because it is more time efficient than analyzing the whole visual field of both eyes. This study investigates the correctness of such an approach by measuring the peripheral wavefront aberrations in both eyes of 22 subjects out to $\pm 40^{\circ}$ horizontally. The largest aberrations (defocus, astigmatism, and coma) were found to be significantly correlated between the left and right eyes when comparing the same temporal or nasal angle. The slope of the regression line was close to \pm 1 (within 0.05 ) for these aberrations, with a negative slope for the horizontally odd aberrations, i.e. the left and right eyes are mirror symmetric. These findings justify that the average result, sampled in one of the two eyes of many subjects, can be generalized to the other eye as well.
\end{abstract}

Keywords: peripheral visual field; off-axis wavefront aberrations; left and right eyes; mirror symmetry

\section{Introduction}

The image on the peripheral retina is often degraded compared with the foveal image, primarily by oblique astigmatism and coma [1-7]. Information on these peripheral aberrations is of interest for research on, for example, the effect of optical errors on peripheral vision [8-11], improving vision for people with central vision field loss [12,13], and eye modeling with individual data retrieved from ocular wavefront tomography $[14,15]$. Furthermore, the interest in measurements of peripheral refractive errors has increased, due to their possible link to myopia [16-18]. One potential problem in peripheral refraction is that the determination of the far-point is not unambiguous. For example, Charman and Atchison have discussed the influence of oblique astigmatism and higher-order aberrations on the best focus value [19]. Therefore, a full wavefront measurement is desired even when only the peripheral refractive state is of interest.

Ideally, data from the whole peripheral visual field of both eyes should be evaluated. However, for reasons of time efficiency, it is common that only a few angles in one of the eyes are sampled. A previous study on foveal aberrations [20] found significant correlations between the left and right eyes for most subjects, with a mean correlation coefficient of 0.517 . The aim of this study is to investigate the correlation of peripheral aberrations over the horizontal meridian between the left and right eyes for different states of accommodation in myopic and emmetropic subjects. The results can give an insight into the overall symmetries that exist between the left and right eyes and justify the prevalent modus operandi of measuring one eye.

\section{Methods}

Peripheral wavefront measurements were performed using two Hartmann-Shack wavefront sensors. These were one laboratory Hartmann-Shack sensor in Murcia, Spain, which has been previously used for peripheral measurements [21,22], and one commercial Hartmann-Shack (COAS-HD-VR) sensor in Kalmar, Sweden, which has been shown to provide repeatable measurements of peripheral aberrations [23]. In total, there were 22 subjects. Informed consent was obtained beforehand, and the study followed the tenets of the Helsinki declaration. The subjects were measured without any refractive correction and with natural pupil size and accommodation (no cycloplegia). With both systems, three measurements per angle were performed and the resulting Zernike coefficients were averaged.

\footnotetext{
*Corresponding author. Email: linda@biox.kth.se
} 


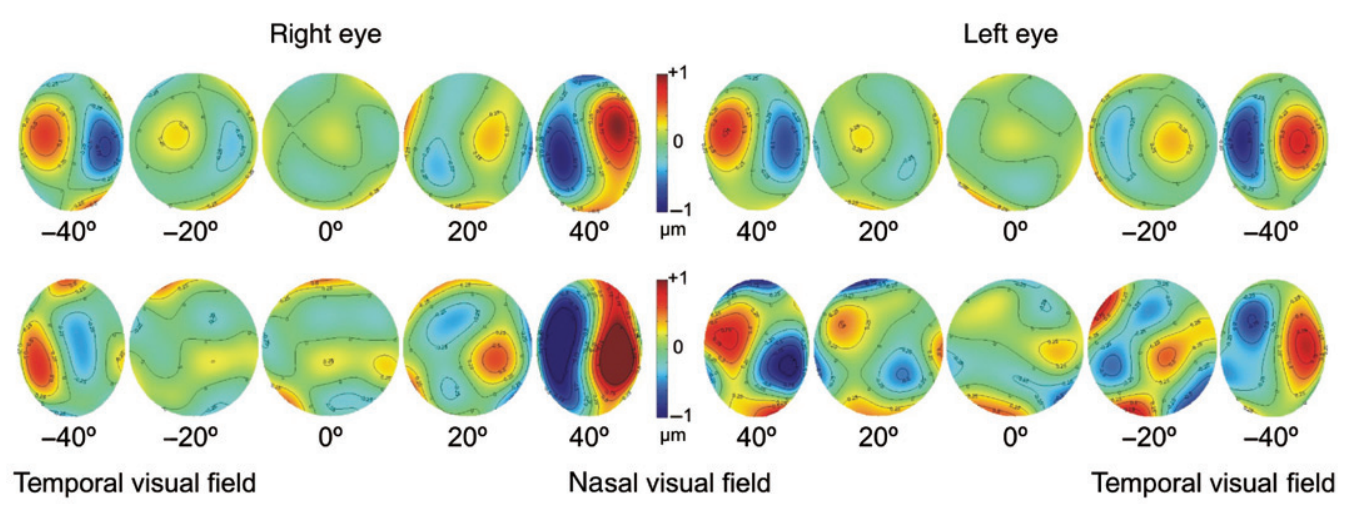

Figure 1. Wavefront maps showing the higher order aberrations in five angles for the left and right eyes of two subjects (an elliptical pupil with major diameter of $4 \mathrm{~mm}$ has been cut out). The upper row shows an example of a subject with a high mirror symmetry between the left and right eyes, whereas the maps on the lower row are from a subject with a low symmetry.

\subsection{Laboratory Hartmann-Shack wavefront sensor}

The peripheral aberrations of the horizontal visual field were measured at $0^{\circ}, \pm 10^{\circ}, \pm 20^{\circ}, \pm 30^{\circ}$, and $\pm 40^{\circ}$ in the left and right eyes of eight subjects (age range: 25-32 years); four emmetropes and four myopes (sphere: $-2.00--7.25 \mathrm{D}$, astigmatism $\leq 0.75 \mathrm{D}$ ). Zernike coefficients up to the fifth order were calculated for a circular wavefront circumscribing the entire elliptical pupil, and then shrunk to $4 \mathrm{~mm}$ diameter [24] to allow comparison. The subjects turned their eyes relative to the Hartmann-Shack sensor. The system had an open field of view and the accommodation was controlled by binocular fixation to a target at a distance of $2 \mathrm{~m}$, except for the two most myopic subjects who used a fixation target at $25 \mathrm{~cm}$ distance instead.

\subsection{COAS-HD-VR}

The peripheral aberrations of the horizontal visual field were measured at $0^{\circ}, \pm 20^{\circ}$, and $\pm 35^{\circ}$ in the left and right eyes of 14 subjects (age range: 22-31 years); seven emmetropes and seven myopes (sphere: $-2.00-$ $-5.00 \mathrm{D}$, astigmatism $\leq 0.50 \mathrm{D}$ ). The standard software package of the instrument was used to calculate the Zernike coefficients up to the fifth order for a circular wavefront with a radius equal to the average of the major and minor axes of the elliptical pupil. The pupil diameter was then shrunk to $4 \mathrm{~mm}$ [24] to allow comparison. The subjects turned their eyes relative to the Hartmann-Shack sensor and fixated through a monocular Badal system to a target with no accommodative demand. Furthermore, the Badal system was used to provide a target with $4 \mathrm{D}$ accommodative demand, for which the peripheral aberrations of the left and right eyes were also measured. During the measurements the other eye was occluded.

\section{Results}

Figure 1 shows an example of the wavefront aberrations measured in two subjects for the horizontal angles of $0^{\circ}, \pm 20^{\circ}$, and $\pm 40^{\circ}$ of the right and left eyes. The refractive errors (i.e. the second-order Zernike coefficients) have been removed to show more clearly the symmetry in the higher order aberrations that exist between the left and right eyes when comparing the same temporal/nasal angle (compare the same angle for the right eye and the left eye). The subjects have been chosen in order to show one subject (upper row) with a high mirror symmetry between the left eye and the right eye and one subject (lower row) with a lower symmetry.

The correlation of peripheral aberrations over the horizontal meridian between the right and left eyes for all subjects was quantified by calculating the correlation and the slope of the best fit regression lines between the two eyes for the second to fourth radial order Zernike coefficients (notation following the ANSI standard [25]). Table 1 gives the slope values for the fovea (denoted by $0^{\circ}$ ) as well as for the off-axis angles of $\pm 10^{\circ}$ (denoted by $10^{\circ}$ ), $\pm 20^{\circ}$ (denoted by $20^{\circ}$ ), and a combination of $\pm 30^{\circ}, \pm 35^{\circ}$, and $\pm 40^{\circ}$ (denoted by $30^{\circ}-40^{\circ}$ ) for each Zernike coefficient separately. The last column shows the best fit slope when data from all angles are taken into consideration. Note that the number of subjects and measurements vary between the columns as the two instruments were measuring in different off-axis angles. The stars indicate that the correlation was statistically significant $(p<0.01)$. The slope values of Table 1 are for all 
Table 1. Slopes of the best fit regression line between the left and right unaccommodated eye for second- to fourth-order Zernike coefficients in different off-axis angles (22 subjects).

\begin{tabular}{lccccc}
\hline & $0^{\circ}$ & $10^{\circ}$ & $20^{\circ}$ & $30^{\circ}-40^{\circ}$ & $0^{\circ}-40^{\circ}$ \\
\hline$c_{2}^{-2}$ & 0.12 & -0.18 & $-0.86^{*}$ & $-1.10^{*}$ & $-0.92^{*}$ \\
$c_{2}^{0}$ & $0.92^{*}$ & $0.91^{*}$ & $0.93^{*}$ & $1.00^{*}$ & $0.96^{*}$ \\
$c_{2}^{2}$ & $0.84^{*}$ & $0.89^{*}$ & $0.96^{*}$ & $1.00^{*}$ & $1.00^{*}$ \\
$c_{3}^{-3}$ & 0.28 & -0.03 & 0.01 & 0.09 & 0.16 \\
$c_{3}^{-1}$ & $0.45^{*}$ & 0.25 & $0.42^{*}$ & 0.30 & $0.35^{*}$ \\
$c_{3}^{1}$ & -0.45 & $-0.58^{*}$ & $-0.95^{*}$ & $-1.00^{*}$ & $-0.99^{*}$ \\
$c_{3}^{3}$ & 0.00 & 0.40 & -0.32 & $-0.81^{*}$ & $-0.51^{*}$ \\
$c_{4}^{-4}$ & 0.18 & 0.20 & -0.18 & 0.00 & -0.03 \\
$c_{4}^{-2}$ & 0.05 & 0.06 & -0.35 & $-0.36^{*}$ & $-0.23^{*}$ \\
$c_{4}^{0}$ & $0.67 *$ & $0.64^{*}$ & $0.73^{*}$ & $0.77^{*}$ & $0.81^{*}$ \\
$c_{4}^{2}$ & 0.23 & $0.68^{*}$ & $0.84^{*}$ & $0.57^{*}$ & $0.69^{*}$ \\
$c_{4}^{4}$ & 0.04 & -0.02 & -0.04 & 0.16 & 0.11 \\
\hline
\end{tabular}

*indicates $p<0.01$.

Table 2. Slopes of the best fit regression line between the left and right eyes for second-to fourth-order Zernike coefficients averaged over all angles (corresponding to the last column of Table 1). The two first columns show the slopes for unaccommodated emmetropes (Em) and myopes (My) with 11 subjects in each group. The last column (Acc) gives the slopes for the 14 subjects who were also measured while accommodating $\left(0^{\circ}-35^{\circ}\right)$.

\begin{tabular}{lccc}
\hline & Em & My & Acc \\
\hline$c_{2}^{-2}$ & $-1.00^{*}$ & $-0.88^{*}$ & $-0.63^{*}$ \\
$c_{2}^{0}$ & $0.90^{*}$ & $0.95^{*}$ & $1.00^{*}$ \\
$c_{2}^{2}$ & $1.07^{*}$ & $1.00^{*}$ & $0.91^{*}$ \\
$c_{3}^{-3}$ & -0.05 & $0.42^{*}$ & $0.57^{*}$ \\
$c_{3}^{-1}$ & 0.14 & $0.61^{*}$ & $0.67^{*}$ \\
$c_{3}^{1}$ & $-1.05^{*}$ & $-0.93^{*}$ & $-1.10^{*}$ \\
$c_{3}^{3}$ & $-0.56^{*}$ & $-0.43^{*}$ & $-0.49^{*}$ \\
$c_{4}^{-4}$ & 0.09 & -0.19 & -0.20 \\
$c_{4}^{-2}$ & -0.04 & $-0.39^{*}$ & $-0.36^{*}$ \\
$c_{4}^{0}$ & $0.70^{*}$ & $0.88^{*}$ & $0.56^{*}$ \\
$c_{4}^{2}$ & $0.55^{*}$ & $0.76^{*}$ & $0.65^{*}$ \\
$c_{4}^{4}$ & -0.09 & 0.23 & 0.27 \\
\hline
\end{tabular}

*indicates $p<0.01$.

unaccommodated eyes, including both emmetropes and myopes. In Table 2, the slopes for all angles are given separately for the emmetropic and the myopic subjects as well as for the subgroup of 14 subjects who were also measured during accommodation with the COAS-HD-VR system.

Both tables show that defocus $\left(c_{2}^{0}\right)$ is highly correlated between the right and left eye for all angles and there is also some correlation for coma $\left(c_{3}^{-1}\right)$ and spherical aberration $\left(c_{4}^{0}\right)$ over the whole horizontal visual field. When going off-axis, the number of significantly correlated coefficients increases and the slopes approach unity. This is especially obvious for the aberrations whose values depend on the off-axis angle, such as, for example, with/againstthe-rule astigmatism $\left(c_{2}^{2}\right)$ and horizontal coma $\left(c_{3}^{1}\right)$; the slopes of the best fit line are very close to unity in offaxis angles and both coefficients increase with eccentricity as predicted by the Seidel theory and as shown in earlier studies [1-7].

A negative sign of the slopes in Tables 1 and 2 tells us that that aberration has a change in sign when comparing the same temporal/nasal angle of the left and right eyes. If the two eyes are mirror symmetric, such a change in sign is expected for the Zernike coefficients that are odd over the horizontal meridian. This is also what Tables 1 and 2 show; the significant slopes for the odd coefficients $c_{2}^{-2}, c_{3}^{1}, c_{3}^{3}, c_{4}^{-4}$, and $c_{4}^{-2}$ are all negative, whereas the other significant slopes are all positive. Consequently, the right and left eyes are mirror symmetric over the horizontal field. The mirror symmetry can also be seen in Figure 2, where the same data as given in Table 1 are pictured with the Zernike coefficients of the right eye plotted against those of the left. The three graphs show defocus, astigmatism, and higher (third-fourth) order aberrations. The blue color is used for aberrations that are even over the horizontal meridian and red for the odd ones. An example of the even variation of astigmatism $\left(c_{2}^{2}\right)$, the odd variation of coma $\left(c_{3}^{1}\right)$, and the small variation in spherical aberration $\left(c_{4}^{0}\right)$ over the horizontal meridian of the right and left eye of one subject is shown in Figure 3. As can be seen, the nasal visual field generally has larger aberrations.

\section{Discussion}

The advantage of using two different instruments, one laboratory and one commercial, with separate analyzing software and located in two different laboratories is that the risk of systematic errors influencing the results is lower. In this study, it is difficult to compare the systems to find any potential bias since different subjects were measured in the different laboratories. However, when analyzing the results separately for the two systems, the slope of the regression line between the left and the right eyes was still close to one for defocus, with/against-the-rule astigmatism, and horizontal coma, although the overall correlation was somewhat weaker with the laboratory HartmannShack sensor.

The instruments also differed in the accommodative stimuli presented to the subjects; the laboratory 

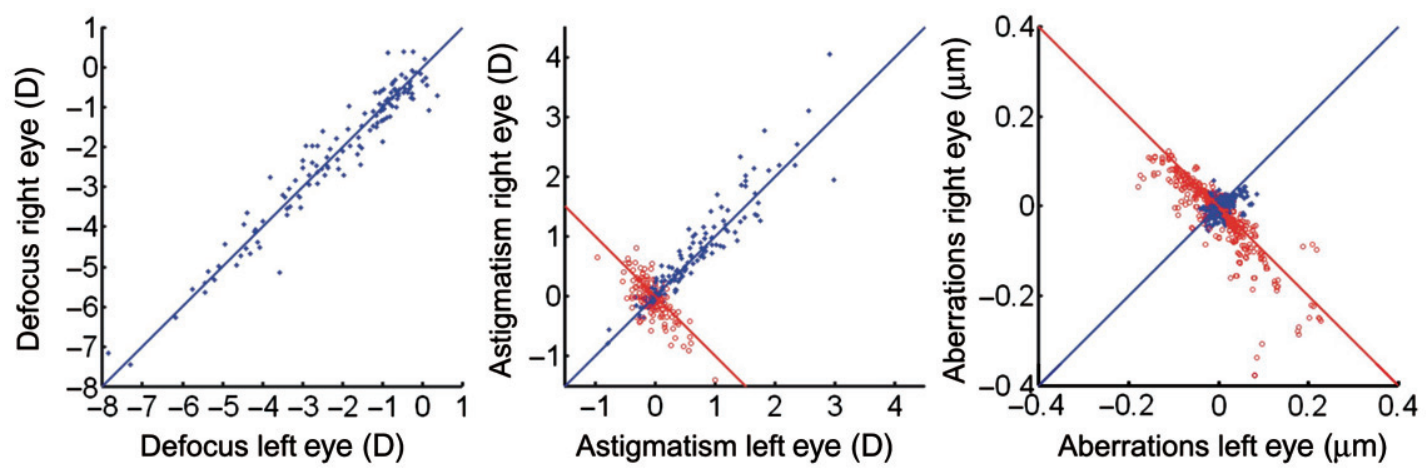

Figure 2. The peripheral aberrations of the right eye plotted against those of the left for all subjects in the study (relaxed accommodation). The two first graphs show defocus and astigmatism in diopters and the third graph shows the third- and fourth-order Zernike coefficients in $\mu \mathrm{m}$ for a $4 \mathrm{~mm}$ pupil. The aberrations that are even over the horizontal meridian are plotted as blue stars and the odd are plotted as red circles. To guide the reader lines are plotted through zero with a slope of +1 (blue) and -1 (red). (The color version of this figure is included in the online version of the journal.)
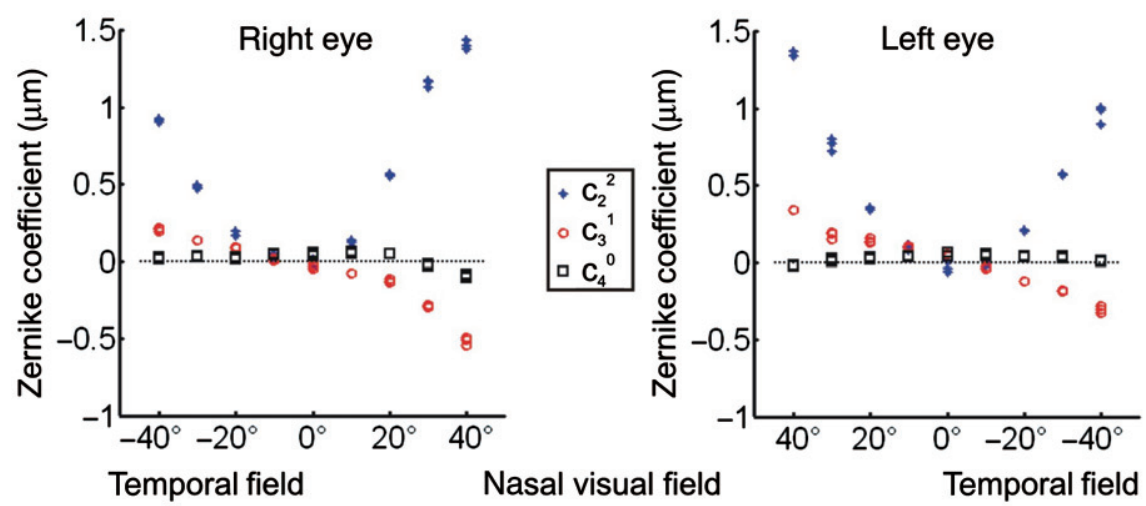

Figure 3. The two graphs show how the with/against-the-rule astigmatism $\left(c_{2}^{2}\right)$, horizontal coma $\left(c_{3}^{1}\right)$, and spherical aberration $\left(c_{4}^{0}\right)$ vary with the off-axis angle in the right and left eyes for one subject (three measurements per angle, pupil diameter of $4 \mathrm{~mm}$ ). (The color version of this figure is included in the online version of the journal.)

sensor had a binocular fixation target with an accommodative demand of $0.5 \mathrm{D}$ for the emmetropes, whereas the COAS-HD-VR was used together with a monocular target seen through a lens placed to give either $0 \mathrm{D}$ or $4 \mathrm{D}$ of accommodative demand. The choice of more than one accommodative demand was to investigate the symmetry for different viewing distances. As expected, we found equal symmetry in all three settings (see the previous paragraph, the last column of Table 1, and the last column of Table 2).

The mirror symmetry between the right and left eyes, which this study found over the whole horizontal meridian, has been noted earlier in a study on foveal aberrations of the right and left eyes [20]; in that study ten out of the 12 second- to fourth-order Zernike coefficients showed a significant correlation between the right and left eyes for the 109 subjects $(p<0.01)$. The fact that only four coefficients in Table 1 showed a significant correlation for the on-axis wavefronts can be explained by the lower number of subjects in the current study. However, the two studies agree on the most correlated aberrations (correlation coefficient of our foveal data compared with the correlation given in [20] in parentheses): defocus $c_{2}^{0}$ with 0.99 (0.98), spherical aberration $c_{4}^{0}$ with 0.79 (0.82), astigmatism $c_{2}^{2}$ with $0.78(0.77)$, and coma $c_{3}^{-1}$ with 0.54 (0.69).

The fact that there is a mirror symmetry between the two eyes also off-axis means that one can adopt the study protocol described in the introduction, i.e. it is enough to sample one of the subjects' two eyes. However, when drawing this general conclusion, it is important to bear in mind that it applies to a population of subjects and that there can be individual differences, as shown by the wavefronts on the lower row of Figure 1. Additionally, the correlation between 
the two eyes over a large part of the horizontal field suggests that the right and left eyes have similar properties, both regarding optics and shape of the retina. This might be one of the reasons why the emmetropization process and the prevalence of myopia are similar in both eyes.

While processing the wavefront data, some correlation was also found between the temporal and nasal fields of the same eye, especially for astigmatism (correlation coefficient 0.75). In spite of that, we recommend cautiousness when only sampling one half of the horizontal meridian, for two reasons. First, defocus showed large differences between individuals in how it varied with the off-axis angle over the temporal and nasal halves, as earlier noted by Gustafsson and co-workers and Ferree and colleagues $[5,26]$. Secondly, the higher-order aberrations tended to be larger in the nasal visual field, which can also be seen for astigmatism in Figure 3 and in the figures of references $[2,3,21,23]$.

Generally, the amount of aberrations and their behavior with the off-axis angle in this study are in agreement with earlier studies [1-7]; i.e. a quadratic increase of astigmatism, close to linear increase of coma, and slightly lower spherical aberration in large off-axis angles. As both emmetropic and myopic subjects were measured in the study we also made some additional analysis of the change in defocus with angle for the two groups separately. The emmetropes were, on average, more myopic in the periphery (relative to the fovea) compared with the myopes as found in many other studies, see, for example, the review by Charman and Radhakrishnan [16]. In agreement with an earlier study by Lundström and colleagues [21], we also found the largest difference between myopes and emmetropes in relative peripheral defocus in the nasal visual field of both eyes. However, the seven myopes and seven emmetropes measured with the COAS-HD-VR system did not show the same systematic difference in how the relative peripheral defocus changed with accommodation as in the previous study.

\section{Conclusion}

This study shows that peripheral optical errors are correlated between the left and right eyes. Significant mirror symmetry between the two eyes was found for nine out of 12 second- to fourth-order Zernike coefficients over the horizontal visual field. The degree of symmetry varies between subjects, but when investigating many subjects, the average results sampled in one of the two eyes can be generalized to the other eye as well.

\section{Acknowledgements}

This work is supported by: the Swedish Agency for Innovation Systems (VINNMER 2008-00992); the Faculty of Natural Sciences and Technology, Linnaeus University, Kalmar, Sweden; the foundation Sparbanksstiftelsen Kronan (COAS-HD-VR); the Ministerio de Educación y Ciencia, Spain (Grants FIS2007-64765, FIS2010-14926, CSD200700033 SAUUL); Fundación Séneca (Region de Murcia, Spain) Grant $4524 /$ GERM/06; and by the European Commission's sixth framework program through the Marie Curie Research Training Network MY EUROPIA (MRTNCT-2006-034021).

\section{References}

[1] Lundström, L.; Gustafsson, J.; Unsbo, P. J. Opt. Soc. Am. A 2009, 26, 2192-2198.

[2] Mathur, A.; Atchison, D.A.; Scott, D.H. Opt. Lett. 2008, 33, 863-865.

[3] Atchison, D.A. J. Biomed. Opt. 2006, 11, 034026.

[4] Seidemann, A.; Schaeffel, F.; Guirao, A.; Lopez-Gil, N.; Artal, P. J. Opt. Soc. Am. A 2002, 19, 2363-2373.

[5] Gustafsson, J.; Terenius, E.; Buchheister, J.; Unsbo, P. Ophthalmic Physiol. Opt. 2001, 21, 393-400.

[6] Guirao, A.; Artal, P. Vision Res. 1999, 39, 207-217.

[7] Navarro, R.; Moreno, E.; Dorronsoro, C. J. Opt. Soc. Am. A 1998, 15, 2522-2529.

[8] Rosén, R.; Lundström, L.; Unsbo, P.; Invest. Ophthalmol. Visual Sci. 2011, 52, 318-323.

[9] Lundström, L.; Manzanera, S.; Prieto, P.M.; Ayala, D.B.; Gorceix, N.; Gustafsson, J.; Unsbo, P.; Artal, P. Opt. Express 2007, 15, 12654-12661.

[10] Wang, Y.Z.; Thibos, L.N.; Bradley, A. Invest. Ophthalmol. Vis Sci. 1997, 38, 2134-2143.

[11] Artal, P.; Derrington, A.M.; Colombo, E. Vision Res. 1995, 35, 939-947.

[12] Lundström, L.; Unsbo, P.; Gustafsson, J. Optom. Vis. Sci. 2007, 84, 1046-1052.

[13] Gustafsson, J.; Unsbo, P. Optom Vis Sci. 2003, 80, 535-541.

[14] Goncharov, A.V.; Nowakowski, M.; Sheehan, M.T.; Dainty, C. Opt. Express 2008, 16, 1692-1703.

[15] Wei, X.; Thibos, L. Opt. Express 2008, 16, 20490-20502.

[16] Charman, W.N.; Radhakrishnan, H. Ophthal. Physiol. Opt. 2010, 30, 321-338.

[17] Mutti, D.O.; Hayes, J.R.; Mitchell, G.L.; Jones, L.A.; Moeschberger, M.L.; Cotter, S.A.; Kleinstein, R.N.; Manny, R.E.; Twelker, J.D.; Zadmik, K.; CLEERE Study Group. Invest. Ophthalmol. Vis Sci. 2007, 48, 2510-2519.

[18] Hoogerheide, J.; Rempt, F.; Hoogenboom, W.P. Ophthalmologica 1971, 163, 209-215.

[19] Charman, W.N.; Atchison, D.A. Ophthal. Physiol. Opt. 2008, 28, 269-276.

[20] Porter, J.; Guirao, A.; Cox, I.G.; Williams, D.R. J. Opt. Soc. Am. A 2001, 18, 1793-1803.

[21] Lundström, L.; Mira-Agudelo, A.; Artal, P. J. Vision 2009, 9, 17. 
[22] Prieto, P.M.; Vargas-Martín, F.; Goelz, S.; Artal, P. J. Opt. Soc. Am. A 2000, 17, 1388-1398.

[23] Baskaran, K.; Theagarayan, B.; Carius, S.; Gustafsson, J. Optom. Vis. Sci. 2010, 87, 751-759.

[24] Lundström, L.; Unsbo, P. J. Opt. Soc. Am. A 2007, 24, 569-577.
[25] American National Standard for Ophthalmics. ANSIZ80.28-2004. Methods for Reporting Optical Aberrations of Eyes; American National Standards Institute: Washington, DC, 2004.

[26] Ferree, C.E.; Rand, G.; Hardy, C. Am. J. Ophthalmol. 1932, $15,513-522$. 